\title{
Emission Spectroscopic Measurements in the Plenum of the NASA IHF ARC JET FACILITY
}

\author{
Michael W. Winter ${ }^{1}$ \\ University Affiliated Research Center UARC, UC Santa Cruz, NASA Ames Research Center, Moffett Field, CA \\ 94035, USA \\ Dinesh K. Prabhu ${ }^{2}$ \\ ELORET Corporation, Sunnyvale, CA, 94086, USA \\ Jaswinder S. Taunk ${ }^{3}$ \\ Jacobs Technology, NASA Ames Research Center, Moffett Field, CA 94035, USA \\ and \\ Imelda Terrazas-Salinas ${ }^{4}$ \\ NASA Ames Research Center, Moffett Field, CA 94035, USA
}

\begin{abstract}
A newly designed segment with optical access was installed in the plenum chamber of the 60 MW Interaction Heating arcjet Facility at NASA Ames Research Center. This special segment has ports located off axis, and the optical fibers can be inserted into these ports. The special segment allows for optical examination of the arc-heated flow as it enters the plenum, and thus assists in determining estimates of the thermodynamic state of the inflow to the convergent section of the nozzle. In the present work, optical emission measurements have been made in VIS-NIR region (wavelengths between $500 \mathrm{~nm}$ to $900 \mathrm{~nm}$ ) for two settings of the arc heater-a $6000 \mathrm{~A}$ condition (high condition) with the minimum amount of radial injection of cold air in the plenum, and a 3300 A condition (low condition) with significant amount of cold air injection to reduce the enthalpy of the arc-heated stream. The results presented here were obtained using an Acton SP300i spectrometer coupled to a Princeton Instruments PI-max intensified camera. In addition to the optical emission measurements, computations were performed for the flow in the plenum and radiation along lines of sight corresponding to the optical ports. Along the centerline, i.e., the longest line of sight across the plenum cross-section, there is good agreement between computations and measurements for the high enthalpy condition, although the off-axis radial profiles show some differences. For the low enthalpy condition, there are significant differences between computations and measurements. The current working hypothesis is that the computational model does not capture details of the mixing process in the plenum.
\end{abstract}

\footnotetext{
${ }^{1}$ Research Scientist, University Affiliated Research Center UARC, UC Santa Cruz, NASA Ames Research Center, Building 230 - Mail Stop 230-3, Moffett Field, CA 94035, Michael.Winter@nasa.gov, AIAA member

${ }^{2}$ Principal Research Scientist, ELORET Corporation, NASA Ames Research Center, Building 230 - Mail Stop 2303, Moffett Field, CA 94035, Associate Fellow AIAA.

${ }^{3}$ Senior Research Engineer, Jacobs Technology, NASA Ames Research Center, Building 229 - Mail Stop 229-4, Moffett Field, CA 94035, AIAA member.

${ }^{4}$ Lead Test Engineer, NASA Ames Research Center, NASA Ames Research Center, Building 229 - Mail Stop 2294, Moffett Field, CA 94035, no AIAA member.
} 


\section{Introduction}

$\mathrm{T}$ HE development and qualification of heatshield materials for high-speed atmospheric entries relies heavily on ground testing in high enthalpy plasma facilities such as the arc jets at NASA Ames Research Center (ARC). Plasma diagnostic methods are necessary to better understand and characterize the test conditions in these facilities. The plenum region between the cathode and the nozzle is of primary interest because the flow conditions in the plenum determine the thermochemical state of the gas mixture expanded through the nozzle into the test section. Furthermore, data measurements in the plenum region are of great value in improving models (primarily inflow conditions) used in numerical simulations or CFD ${ }^{1}$.

Optical methods are particularly well-suited for this task because they do not modify the plasma flow. A set-up for optically probing the flow in the plenum of $60 \mathrm{MW}$ Interaction Heating Facility (IHF) has been developed and applied in the present work. ${ }^{2,3}$

The operational aspects of the IHF are also of interest because enthalpy levels can be tailored using radial injection of cold air to the arc-heated stream. For typical high-enthalpy tests, the amount of additional cold air injected in the plenum is kept to a minimum, or none at all. However, for low-enthalpy tests, the amount of additional cold air is considerably higher, and often higher than that of the primary arc-heated stream. The mixing of the cold and hot streams of air is not well understood, and optically probing the flow in the plenum might provide some insights into this process.

The objectives of the present work were:

1. To obtain calibrated emission signatures of the arc-heated air in the plenum region for various flow rates (with and without additional air injection) and heater settings in order to gain better understanding of the mixing processes of the primary arc-heated stream and the injected secondary air stream.

2. To extract radial variation of the thermal field to guide construction of inflow profiles into the convergentdivergent nozzle.

In order to accomplish these objectives, a combination of experiment and theory was used. Although in general the two approaches are independent, their combination is useful in interpreting measured data, understanding uncertainties, and development of improved models for arc-heated flows expanded through convergent-divergent nozzles.

\section{Facility and Optical Set-up}

The 60 MW Interaction Heating Facility (IHF), one of several arc jet facilities at NASA Ames Research Center, is used to test and qualify spacecraft thermal protection materials in "flight-like" aerothermal environments. ${ }^{4}$ The IHF heater is a constricted arc design with an upstream anode, and a downstream cathode, both consisting of 8 electrode disks. The working gases are injected throughout the arc column established between the anode and the cathode, and are heated to very high temperatures The magnitude of the temperatures achieved, hence the flow energy, depends on the input current, voltage, and flow rates. Following the cathode, there are 4 disks/segments through which additional room temperature air (in the following referred to as "add air") can be injected to tailor the enthalpy of the working medium to the desired test value. This set of 4 segments is referred to as the "plenum" in the present work. The working gas, of appropriate enthalpy, is then expanded through a convergent-divergent nozzle, and the test article is placed in the free jet. Figure 1 shows a sketch of the IHF design.

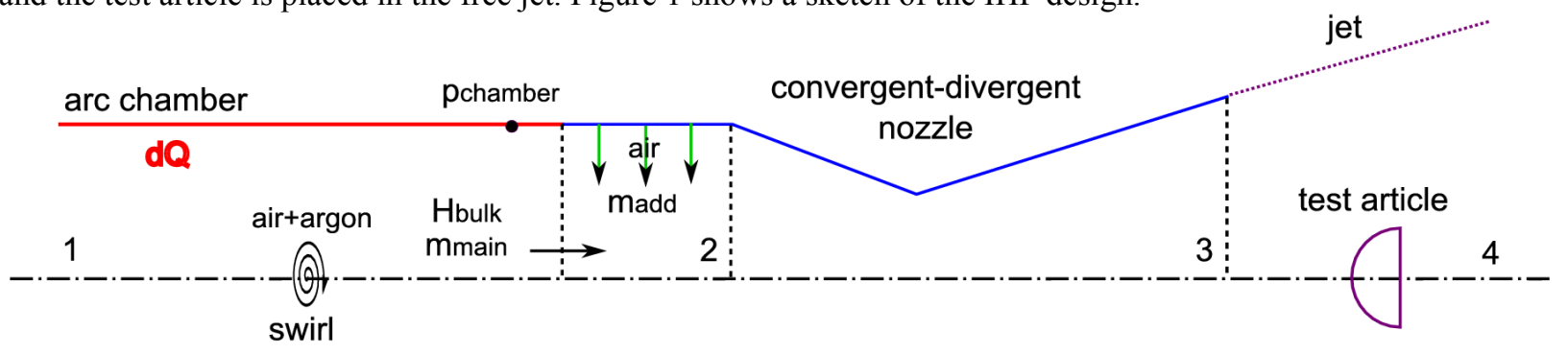

Figure 1. Sketch of the IHF working principle.

The operating conditions of the facility are mainly characterized by arc current and voltage, and the different mass flows (main air, add air and argon) yielding a chamber pressure and a bulk enthalpy derived from the balance of input power and cooling losses. For the present work, two conditions, one at high power with minimum add air (high enthalpy) and one at medium power with high add air (low enthalpy) were investigated. The arc heater settings are given in Table 1. 
Table 1. Arc heater settings for the test conditions.

\begin{tabular}{|c|c|c|c|c|c|c|c|}
\hline Condition & $\begin{array}{c}\text { Arc } \\
\text { Current } \\
(\mathbf{A})\end{array}$ & $\begin{array}{c}\text { Arc } \\
\text { Voltage } \\
\text { (V) }\end{array}$ & $\begin{array}{c}\text { Main-Air Mass } \\
\text { Flow Rate } \\
\text { (g/sec) }\end{array}$ & $\begin{array}{c}\text { Add-Air Mass } \\
\text { Flow Rate } \\
\text { (g/sec) }\end{array}$ & $\begin{array}{c}\text { Argon Mass } \\
\text { Flow Rate } \\
\text { (g/sec) }\end{array}$ & $\begin{array}{c}\text { Chamber } \\
\text { Pressure } \\
\text { (kPa) }\end{array}$ & $\begin{array}{c}\text { Bulk } \\
\text { Enthalpy } \\
\text { (MJ/kg) }\end{array}$ \\
\hline Low & 6000 & 7300 & 740 & 55 & 54 & 886 & 21.6 \\
\hline High & 3300 & 6700 & 510 & 740 & 54 & 900 & 8.4 \\
\hline
\end{tabular}

Since the plenum region is not amenable to intrusive measurement/diagnostic techniques, optical methods have to be used. Therefore, between the add air disks and the convergent part of the nozzle, a spacer disk with optical access was inserted. The water-cooled disk used in the present work is an advanced design of the one used for optical measurements in the plenum region of the Aerodynamic Heating facility (AHF) ${ }^{2,3}$. Figure 2 illustrates the geometric configuration of this disk with optical access. The disk has 7 access ports at different cross sections, three of them being at the west side of the facility and four located at the east side. For safety reasons, the heater section is not accessible during testing. To feed the optical signals out of this closed environment, optical fibers have to be used. Due to the geometry of the facility, the minimal fiber lengths had to be $3.5 \mathrm{~m}$ and $4.5 \mathrm{~m}$ for the west and east side access points, respectively. The 7 ports are useful in the reconstruction of radial distributions based on measured emission signatures. For the centerline, two additional ports perpendicular to the above mentioned access points are also available. Each port carries an optical insert, and each insert is an assembly of a sapphire window, an O-ring, a spacer to protect the window, and a backing screw to provide a sealing force. The entire assembly is contained in a cage that can be threaded into the copper segment. A key feature of the design of the optical access disk is the ability to mount and extract the optical port as a single entity. An extension tube with a SMA connector for optical fibers is threaded into the optical port to improve accessibility to the segment, and provide a collimated beam geometry through an aperture of the same size as the $0.4 \mathrm{~mm}$ optical fibers located at the inner end of the extension.

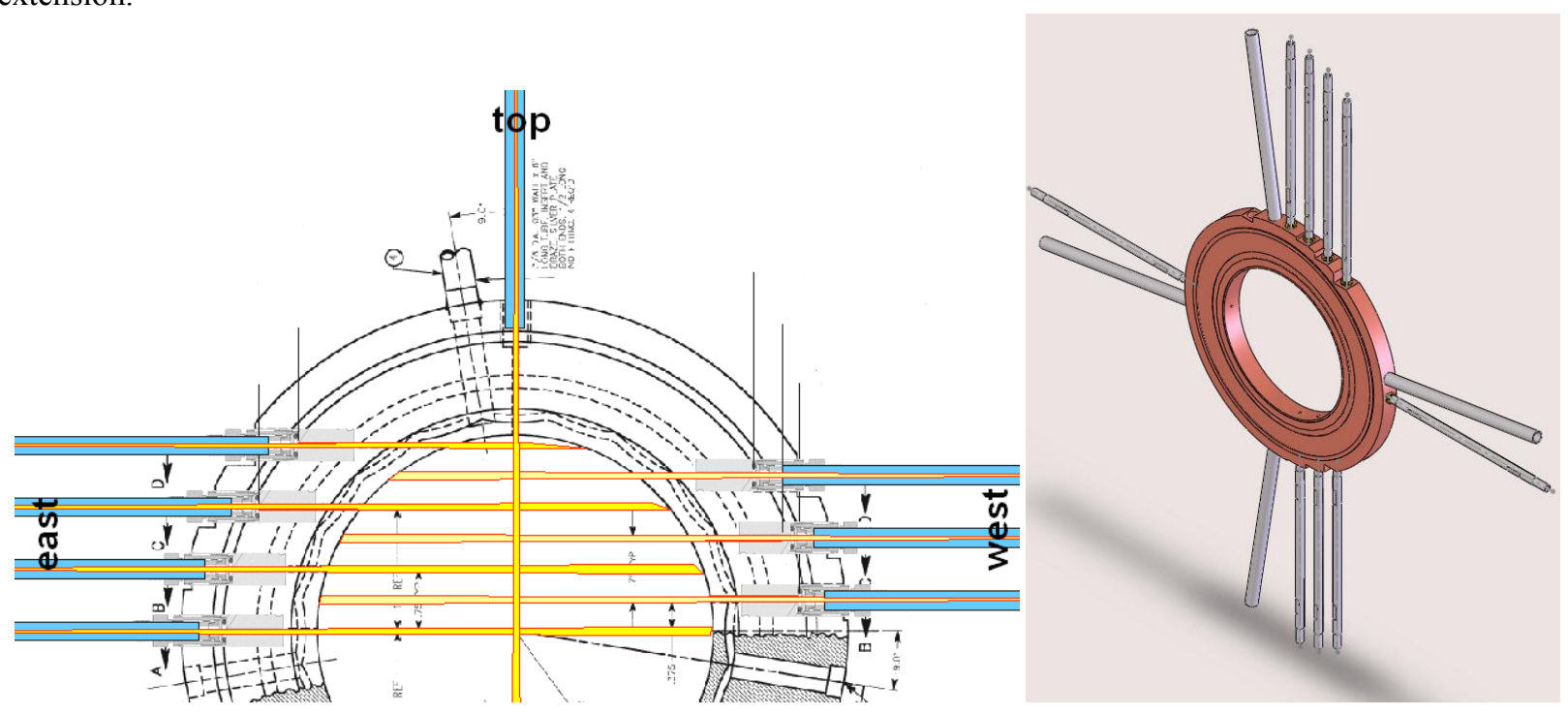

Figure 2. Illustration of the geometrical configuration of the optical plenum disk. 

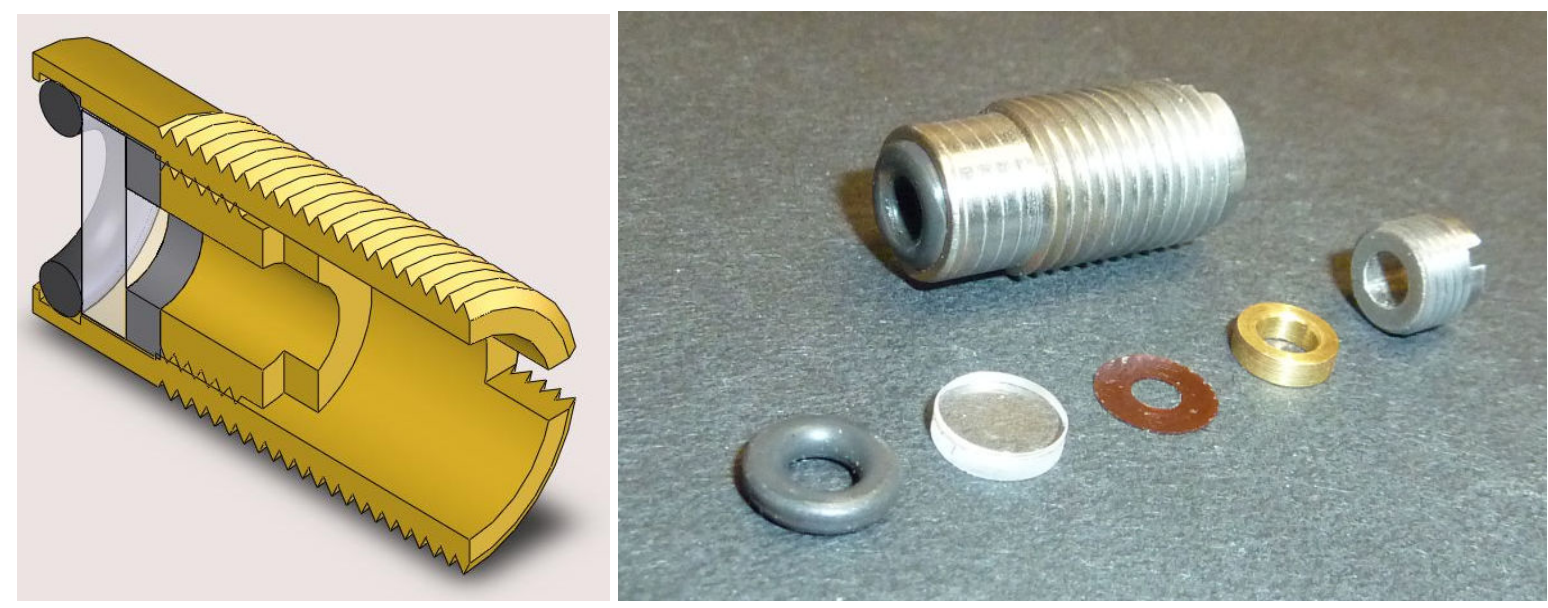

Figure 3. Design of the optical plug unit.

Different spectrometers have been used during the development of the set-up. Initially, several Ocean Optics S2000 and an Echelle ME200 spectrometers were chosen to cover a wavelength range between $200 \mathrm{~nm}$ to $1000 \mathrm{~nm}$. The Echelle ME200 system was used for high spectral resolution $(0.02 \mathrm{~nm})$ across the entire wavelength range. Due to the length of the optical fibers, the transmission of the optical fibers in the UV decreased drastically and signal levels in the UV were weak. Therefore, a useful absolute calibration of the measurements was possible only above $500 \mathrm{~nm}$. To make measurements with all 7 channels, the fibers had to be switched during the test. This caused unnecessarily long test times and introduced additional uncertainties due to the time difference between the single measurements. Therefore, the spectrometric system was changed to an Acton SP300i and an intensified Princeton PI-max camera. To feed all fibers simultaneously into the spectrometer, a customized fiber coupler, which combines the seven single fiber legs into a linear array, was used. Each leg consisted of a fiber bundle of seven individual fibers. The single channels were then imaged on to different vertical positions on the CCD array. This approach also allows for time-resolved measurements to be made to investigate the temporal stability of plasma flows in the plenum of the IHF. However, to achieve sufficient imaging of the fibers on the CCD array, the imaging mirror of the spectrometer had to be masked to about $1 / 4$ of its original size to eliminate off-axis aberration in the SP300i. Figure 4 shows the resulting image of the fiber configuration on the CCD for a typical spectrum.

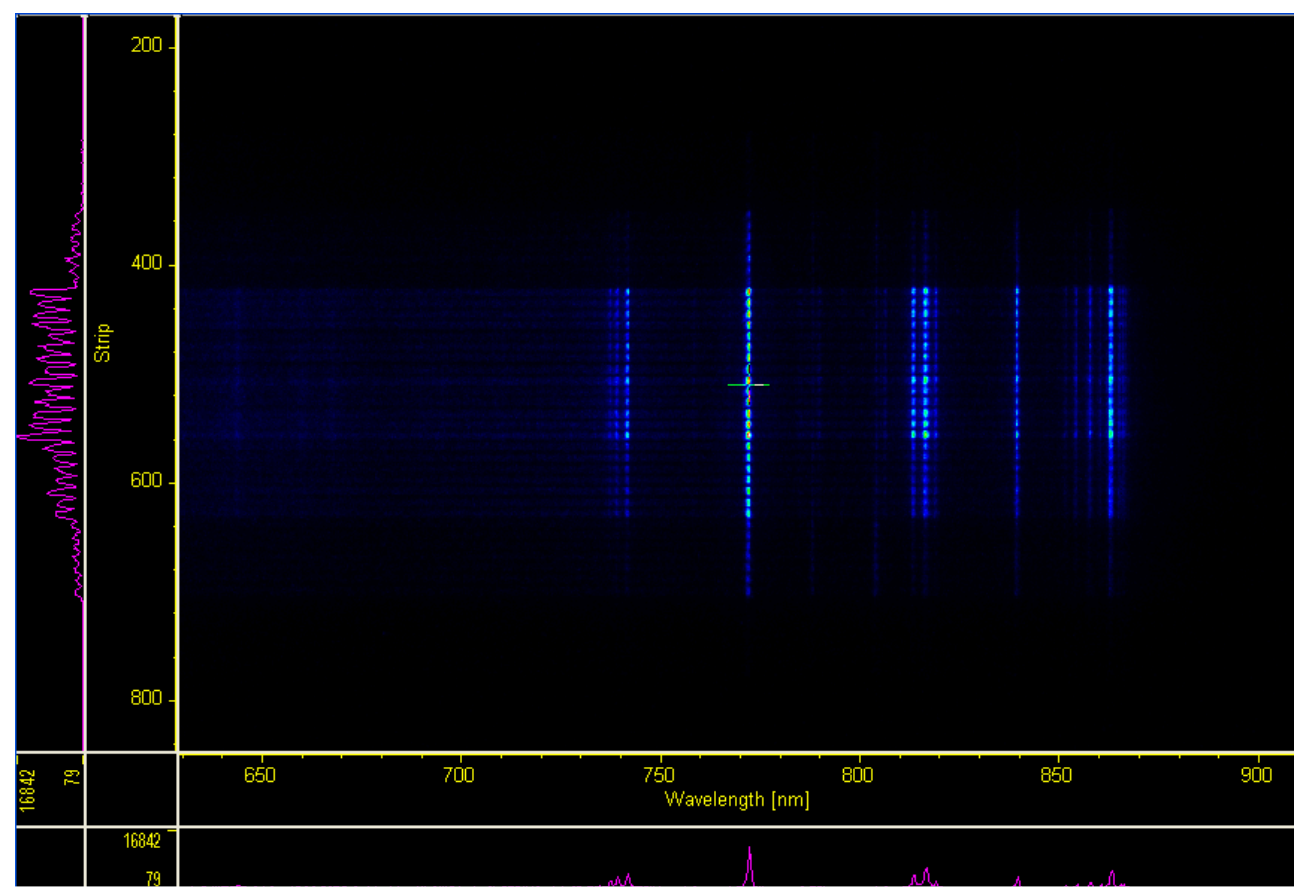

Figure 4. Raw data of a typical plasma showing the coupling of the fibers to spectrometer and CCD.

4

American Institute of Aeronautics and Astronautics 


\section{Data reduction}

In order to optimize the efficiency of the set-up and increase the dynamic range, the fibers in each leg can be integrated in the y-direction of the CCD corresponding to the elongation of each leg in the entrance slit plane which yields the total signal of the corresponding channel. However, the order of magnitude of the measured data varies substantially between the different channels and charge bleeding can occur from the channels in the middle of the segment towards higher off sets to the center line. Therefore, in the present study, only the inner 5 fibers in each leg have been used for the integration.

The measurements were calibrated to absolute spectral radiance units using a calibrated integrating sphere. For the calibration, a spare optical segment was cut in two halves to be able to place the calibration lamp on the axis. Initial ray tracing analysis of the optical path indicated that the sensitivity along the line of sight should be constant. However, measurements with the calibration lamp at different positions on the line of sight showed a small variation of the measured signal on the order of $2 \%$. Further analysis is ongoing. Figure 5 shows the calibration set-up with the calibration lamp.

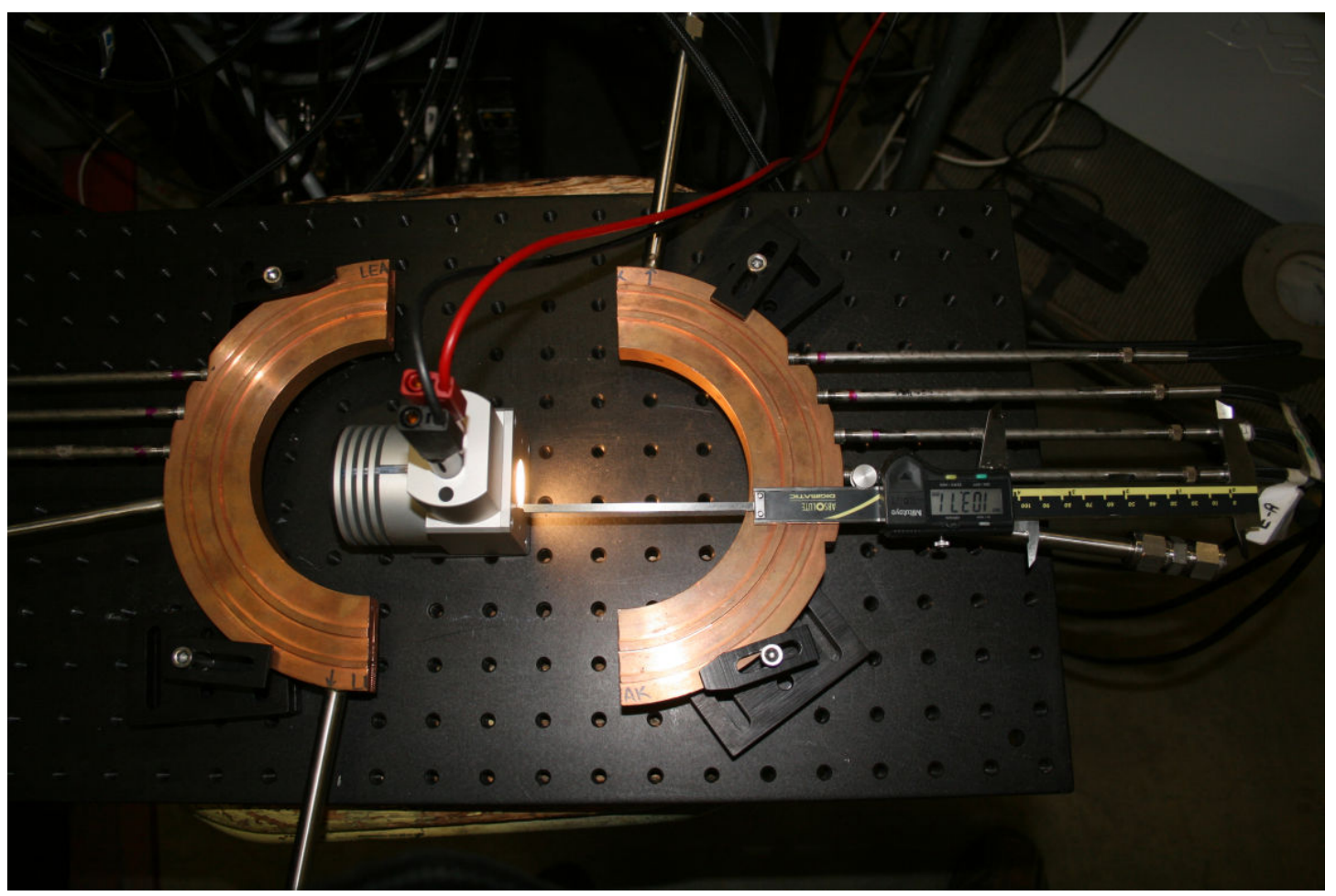

Figure 5. Calibration set-up with the open optical heater segment.

The calibration to absolute radiance was done by building the ratio of measured calibration lamp radiation and theoretical radiance given by the manufacturer. For calibration and plasma measurements, the intensifier gain was kept constant but the acquisition time had to be significantly different between plasma measurement (20ms) and calibration lamp (30s). However, in this range, the camera signal is verified to be sufficiently linear with acquisition time. Since the fibers cut off signals below $500 \mathrm{~nm}$, second order radiation does not have to be accounted for. The calibrated values for the lamp and the overall sensitivity of the set-up on the center line are given in Fig. 6 . The two different response curves originate from geometrical effects inside the spectrometer for different grating positions. However, the system sensitivity is different for each optical port due to the different lengths of the access hole in the copper segment. 


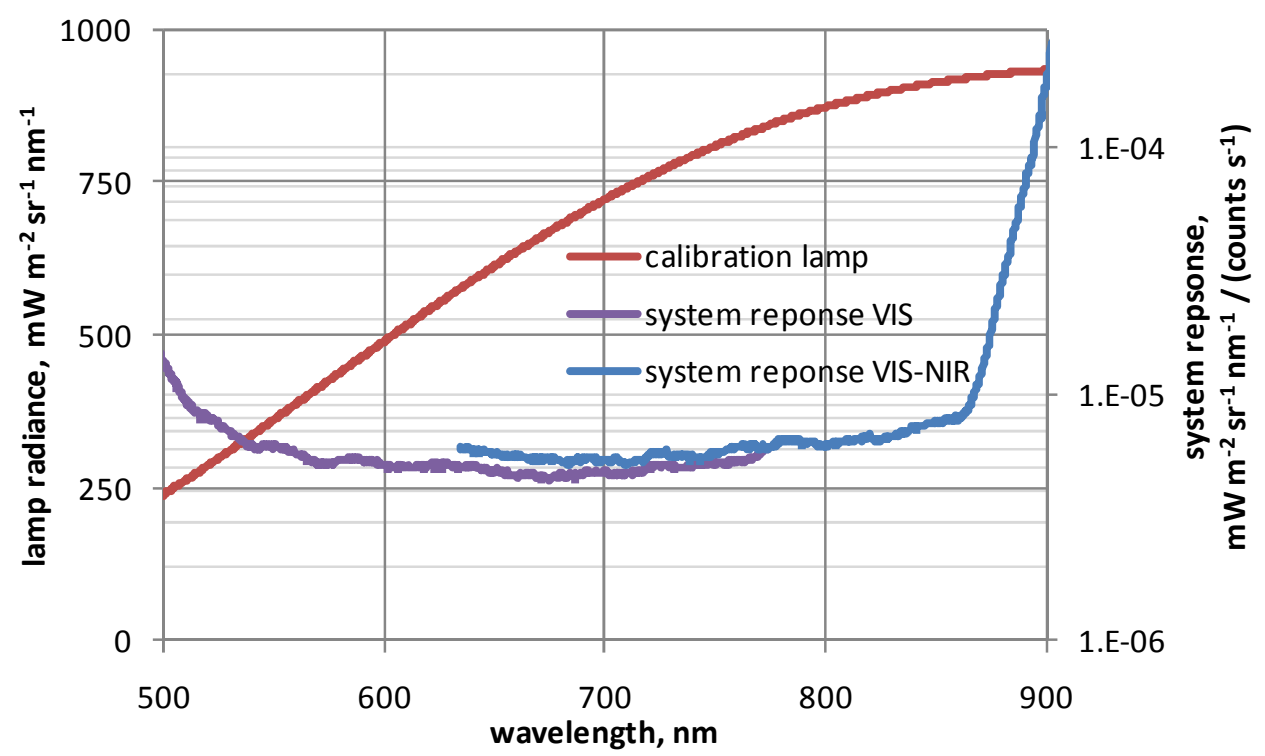

Figure 6. Calibration lamp radiance and system response for the two wavelength ranges with the calibration lamp positioned on the segment axis.

\section{Theory/Modeling}

The procedure for computing flow fields in the plenum, through the nozzle, and into the test chamber are based on the work of Prabhu et al. ${ }^{1}$ Briefly, using the measured flow rates and arc-column pressure, the bulk enthalpy of the flow is estimated. Profiles of enthalpy and mass flux are constructed based on distributions inferred from sweeps of the free jet using nullpoint calorimeters - the underlying assumption is that the flow is adiabatic (i.e., lossless) in the inviscid core flow between the plenum and the test chamber. Flow computations are performed using v3.05 of the in-house code DPLR ${ }^{5}$ assuming a 12 -species $\left(\mathrm{N}_{2}, \mathrm{O}_{2}, \mathrm{NO}, \mathrm{NO}^{+}, \mathrm{N}_{2}{ }^{+}, \mathrm{O}_{2}{ }^{+}, \mathrm{N}, \mathrm{O}, \mathrm{N}^{+}, \mathrm{O}^{+}, \mathrm{Ar}\right.$, and $\left.\mathrm{e}^{-}\right)$air in thermochemical nonequilibrium. It should be noted here that the inflow profiles are constructed assuming that local thermodynamic equilibrium applies at every radial point in the plenum cross-section; the assumption being justified by the fact that the arc-column pressure that develop are greater than $1 \mathrm{~atm}$ in most cases.

From the CFD solutions, profiles of the translational/rotational and vibro-electronic temperatures, and number densities of all the constituent species are extracted at the geometrical positions covered by the optical ports. The extracted profiles are considered to be the simulated lines of sight, and the data are used in the latest version of NEQAIR . ${ }^{6}$ NEQAIR is a line-by-line spectroscopic code with one-dimensional transport of radiative intensity. The principal molecular band systems considered are the $1^{\text {st }}$ and $2^{\text {nd }}$ positive systems of $\mathrm{N}_{2}$, the $1^{\text {st }}$ negative system of $\mathrm{N}_{2}{ }^{+}$, the $\gamma, \beta, \delta$, and $\varepsilon$ systems of NO, the Schumann-Runge system of $\mathrm{O}_{2}$, and the atomic lines of $\mathrm{N}$ and $\mathrm{O}$. Highresolution spectra computed using NEQAIR for these band and line systems are then integrated along the lines of sight taking absorption into account, and convolved with the experimentally determined instrument function to produce synthetic spectra for comparison against the observations.

Figures 7 and 8 illustrate the radial profiles of particle densities (in parts $/ \mathrm{m}^{3}$ ) and temperatures in the plenum (equivalent to the conditions along the line of sight at the center) for both conditions as obtained from the CFD solution. At the high enthalpy condition temperatures between $7000 \mathrm{~K}$ and $8000 \mathrm{~K}$ are obtained. Oxygen $\left(\mathrm{O}_{2}\right)$ is almost fully dissociated over the whole cross section, nitrogen dissociation reaches about $70 \%$ at the center, and ionization reaches a maximum of roughly $0.1 \%$, the main ion being $\mathrm{NO}^{+}$. At the low condition, temperatures range between $4000 \mathrm{~K}$ and $6000 \mathrm{~K}$. Oxygen $\left(\mathrm{O}_{2}\right)$ is still almost fully dissociated in the center, nitrogen dissociation drops down to a maximum of about $13.6 \%$ at the center, and ionization is very weak $(<0.02 \%)$. 


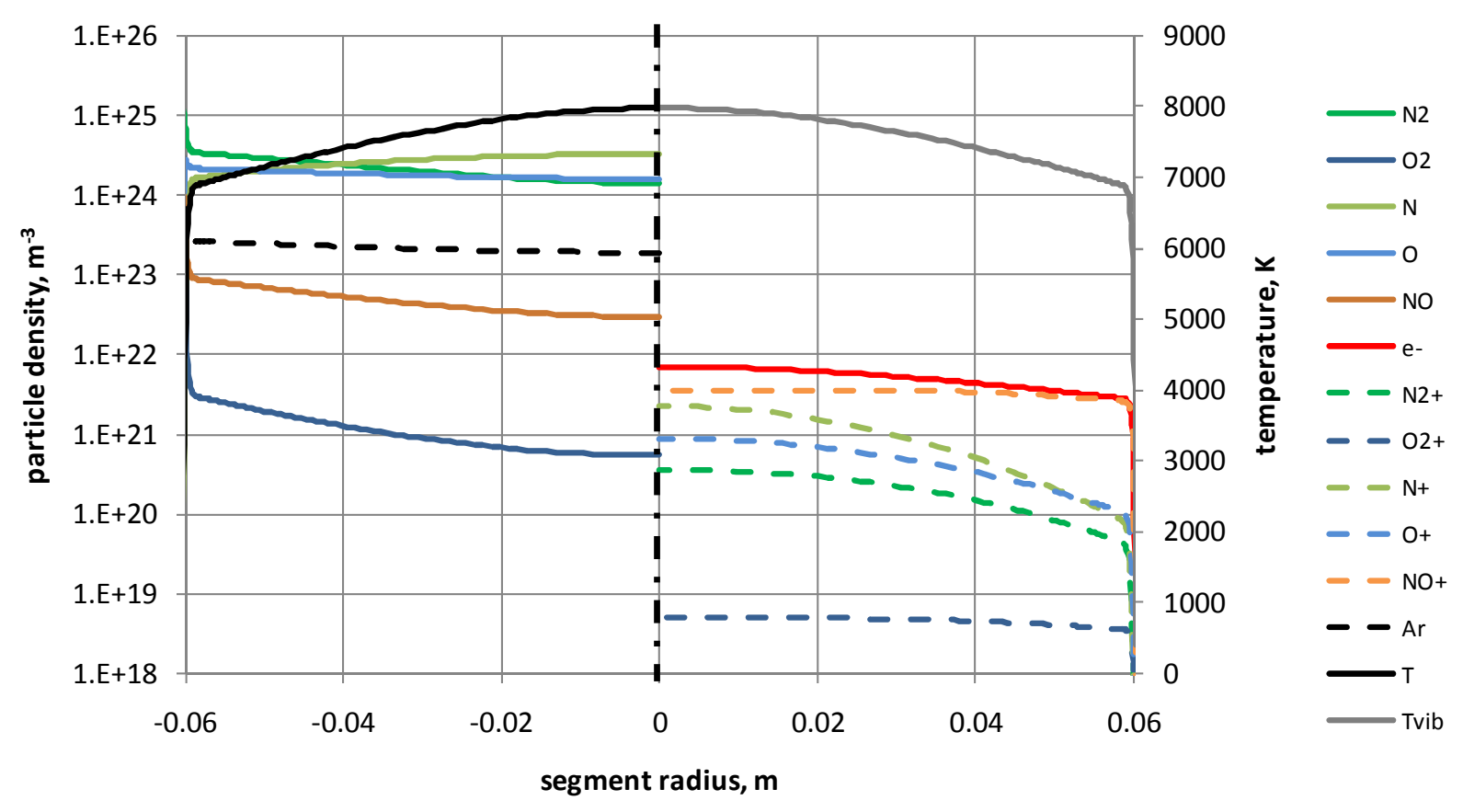

Figure 7. Simulated temperatures and particle densities (neutrals left, ions right) along the centerline in the IHF plenum region for the high enthalpy condition.

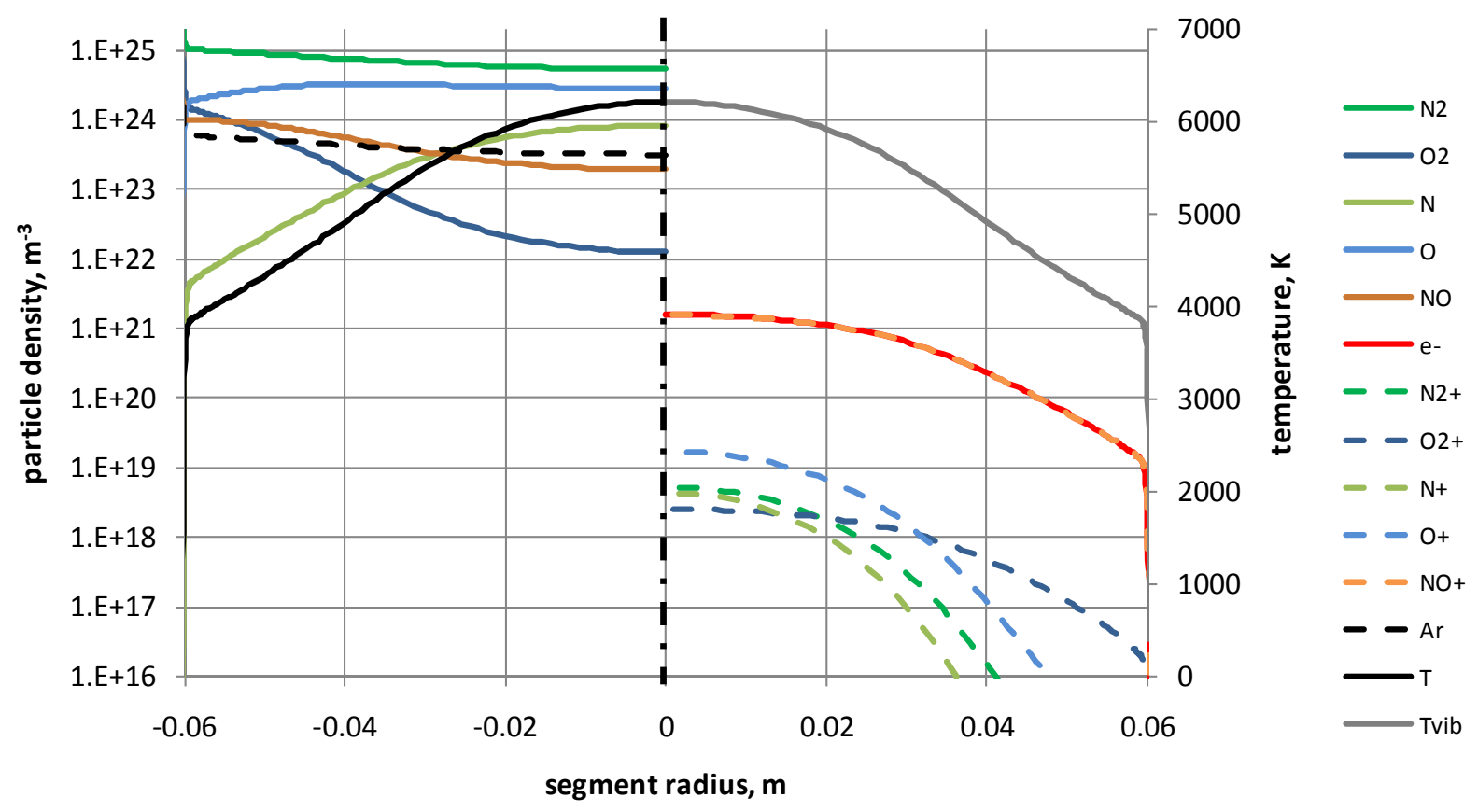

Figure 8. Simulated temperatures and particle densities (neutrals left, ions right) along the centerline in the IHF plenum region for the medium enthalpy condition. 


\section{A. Comparison of experimental and theoretical spectra}

The main radiation is seen from the atomic lines of nitrogen and oxygen. The molecular emission of the $\mathrm{N}_{2} 1^{\text {st }}$ Pos. system is expectedly weak. Continuum radiation is most probably present but has not yet been analyzed. For the line of sight through the center of the optical segment (in the following called center line), the CFD based spectra are in rather good qualitative and quantitative agreement with the measured ones as shown in Fig. 9. However, the ratio of oxygen to nitrogen lines is slightly higher in the CFD based spectra than in the measured ones.

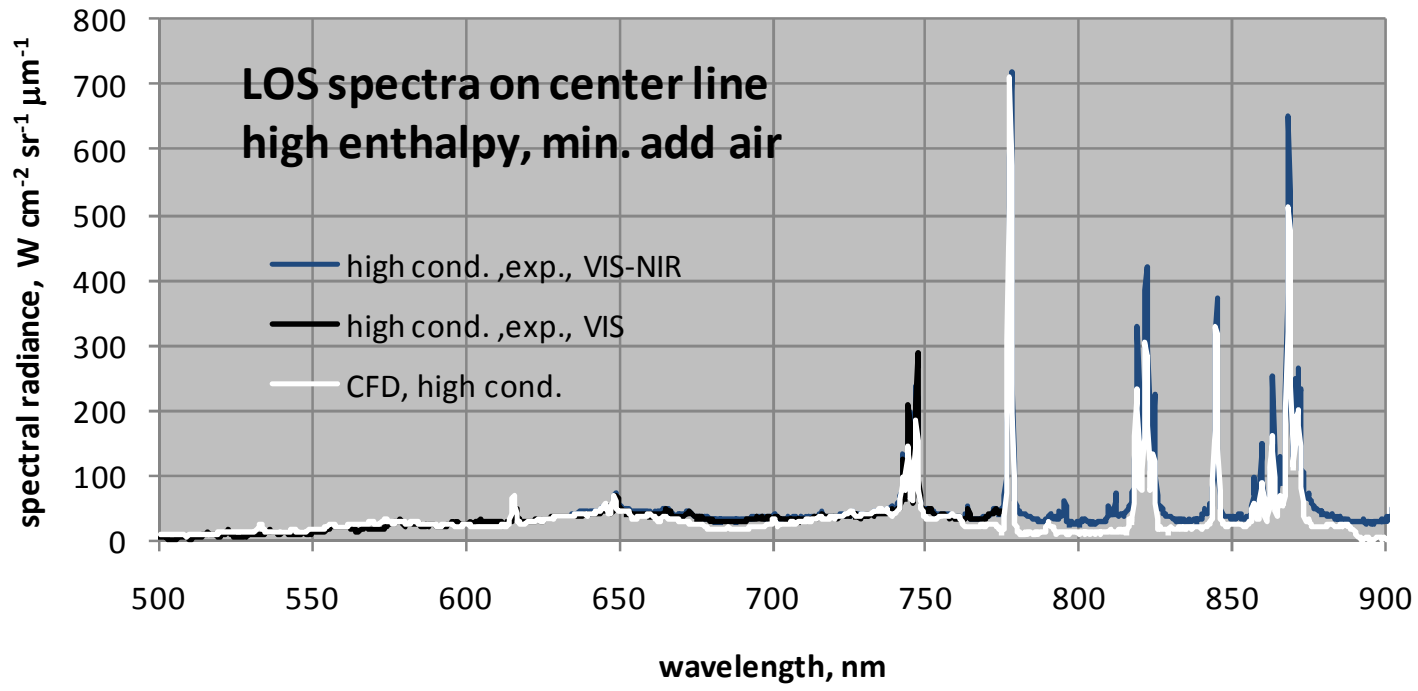

Figure 9. Simulated and measured emission spectra between $500 \mathrm{~nm}$ and $900 \mathrm{~nm}$ on the center line at the high enthalpy condition. The simulated spectra were scaled down to $70 \%$.

Since the atomic lines are the major radiators, the intensities of the oxygen lines at $777 \mathrm{~nm}$ and $845 \mathrm{~nm}$ and the nitrogen lines at $745 \mathrm{~nm}$ and around $820 \mathrm{~nm}$ were spectrally integrated (in the following this integral is called line intensity) for an analysis of the radial distribution. Using the integrated intensity rather than the peak intensity accounts for possible differences of the line shape in experiment and simulation. Trapezoids defined by the used integration limits were used to account for underlying continuum and molecule emission in the experimental data. Separate simulations with only oxygen or nitrogen as radiating species were used to determine the theoretical line emission.

The experimental profiles as presented in Fig. 10 show a concentration of the radiation in the center of the plenum. The maximum at 0.375 inches distance to the axis could be an influence of the shape of the electrical discharge attaching to the last electrode upstream of the optical segment. Apart from that maximum, the CFD based radial distribution is at least close to the measured one in the core flow region but shows a broader profile with atomic line radiation being about 2 times stronger than the experiment at 1.5 inches distance to the middle axis. To compensate for these factors, an decrease in electronic excitation temperature of about $400 \mathrm{~K}$ to $900 \mathrm{~K}$ at high radial positions would be required if the chemistry would be kept constant. Further analysis will include a systematic variation of the CFD plenum condition in terms of the enthalpy profile and potentially also its bulk value to find a matching configuration. Presently. the measurements indicate that the

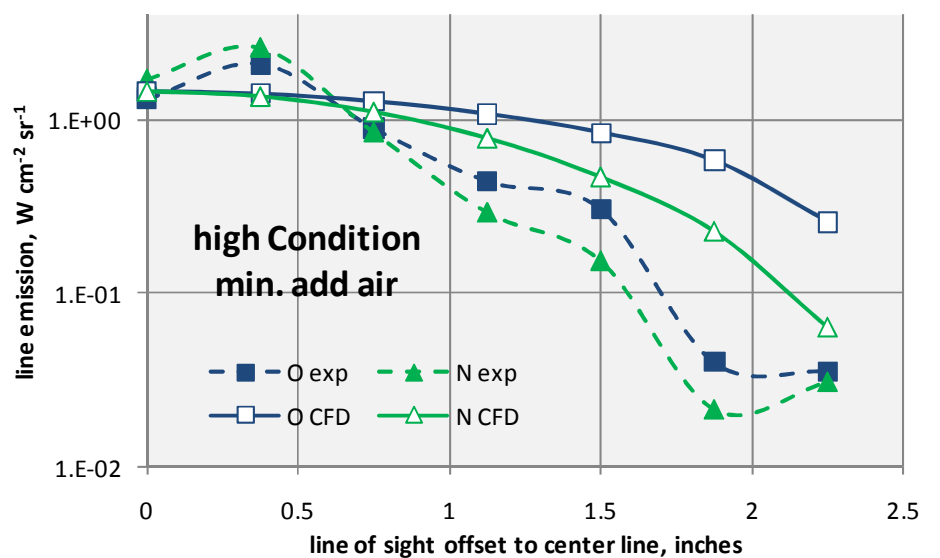

Figure 10. Predicted and measured radial profiles of $O$ and $N$ line intensities at the high enthalpy condition vs. line of sight offset from center line. 
enthalpy outside the core flow is somewhat lower than assumed in the CFD computation. This might be an influence of the small amount of add air.

For the low enthalpy condition, the measured spectra were substantially different from the CFD based ones. At the center line, the simulated lines of atomic oxygen were lower by a factor of about 30 in oxygen line intensity, the nitrogen lines were even weaker in the simulation although clearly seen in the experiment. This clearly indicates that the mixing process of the room temperature add air was not complete (at least not at the plenum position) and therefore did not lower the enthalpy as much as expected from the numerical simulation.

The radial profiles of the line intensities of $\mathrm{O}$ and $\mathrm{N}$ as shown in Fig. 12 appeared qualitatively similar close to the center line, but the measured line emission became higher by several orders of magnitude close to the wall.

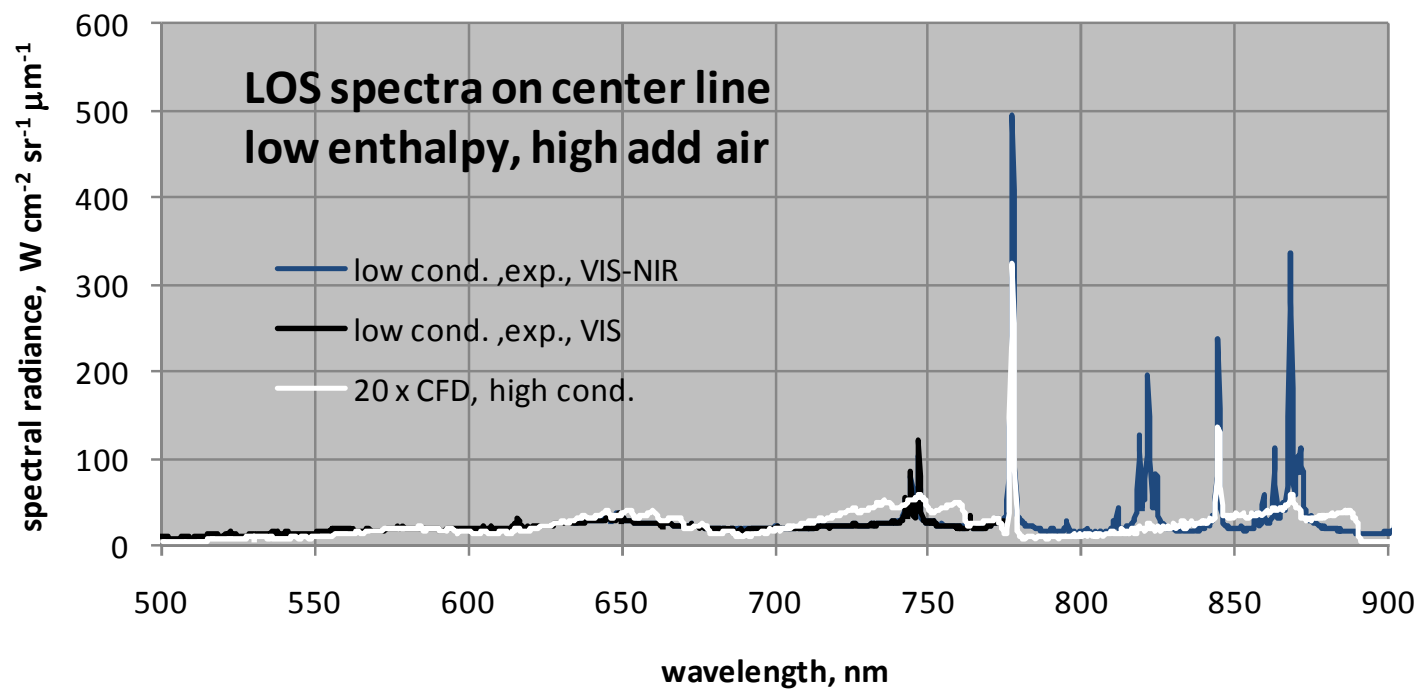

Figure 11. Simulated and measured emission spectra between $500 \mathrm{~nm}$ and $90 \mathrm{~nm}$ on the center line at the low enthalpy condition. The simulated spectra were multiplied by 10 .

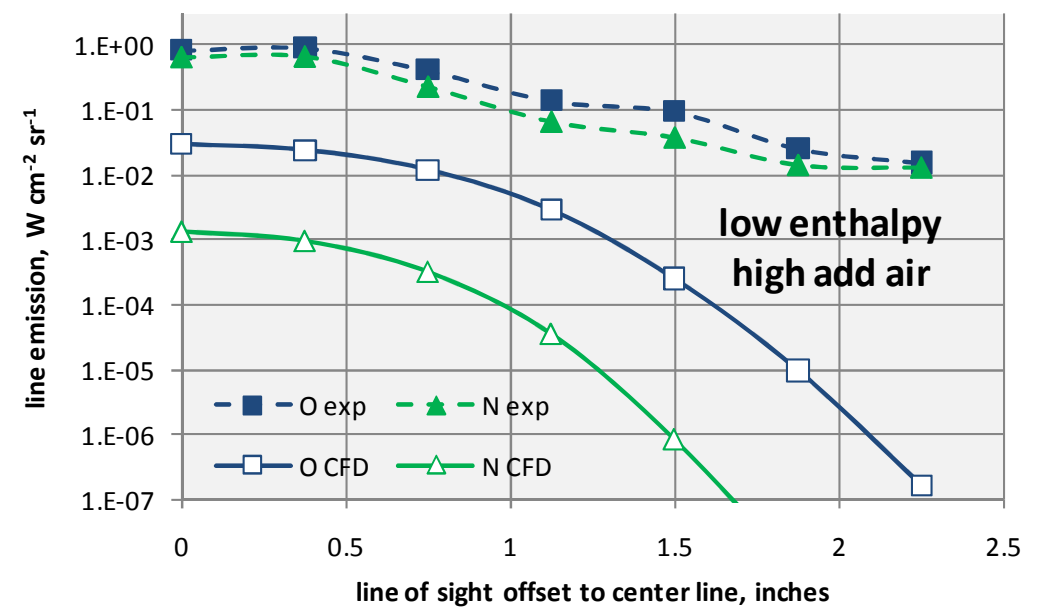

Figure 12. Predicted and measured radial profiles of $O$ and $N$ line intensities at the low enthalpy condition vs. line of sight offset from center line.

If the difference is interpreted as an under-prediction of equilibrium temperature, a modification in the start condition for the CFD analysis will yield both a change in nitrogen particle densities due to chemistry effects and an increase of radiation due to a higher upper state population. Oxygen, however, is already almost fully dissociated so the expected change in chemistry will be low. Therefore, the difference between measured and predicted radiation can be interpreted as an upper limit for the necessary modification of equilibrium temperature to match the experimental data. On the centerline, the measured intensities are roughly 16 times higher than predictions, implying a temperature increase of about $1200 \mathrm{~K}$ from $6200 \mathrm{~K}$ to $7400 \mathrm{~K}$. If this computation is conducted for all radial 
positions, a corrected equilibrium temperature can be derived as shown in Fig. 13. For the computation, the degree of dissociation of oxygen was artificially increased to 1 (assuming that the new temperatures would be high enough to provide full oxygen dissociation) by reducing the ratio between measured and predicted line intensities. The necessary temperature differences are between about $1600 \mathrm{~K}$ up to $2100 \mathrm{~K}$ close to the wall. These results are considered to be preliminary. Of course, a quantitative interpretation of the data will require a more thorough variation in the starting condition for the CFD solution, which unfortunately, was not available at the time of writing the present paper.
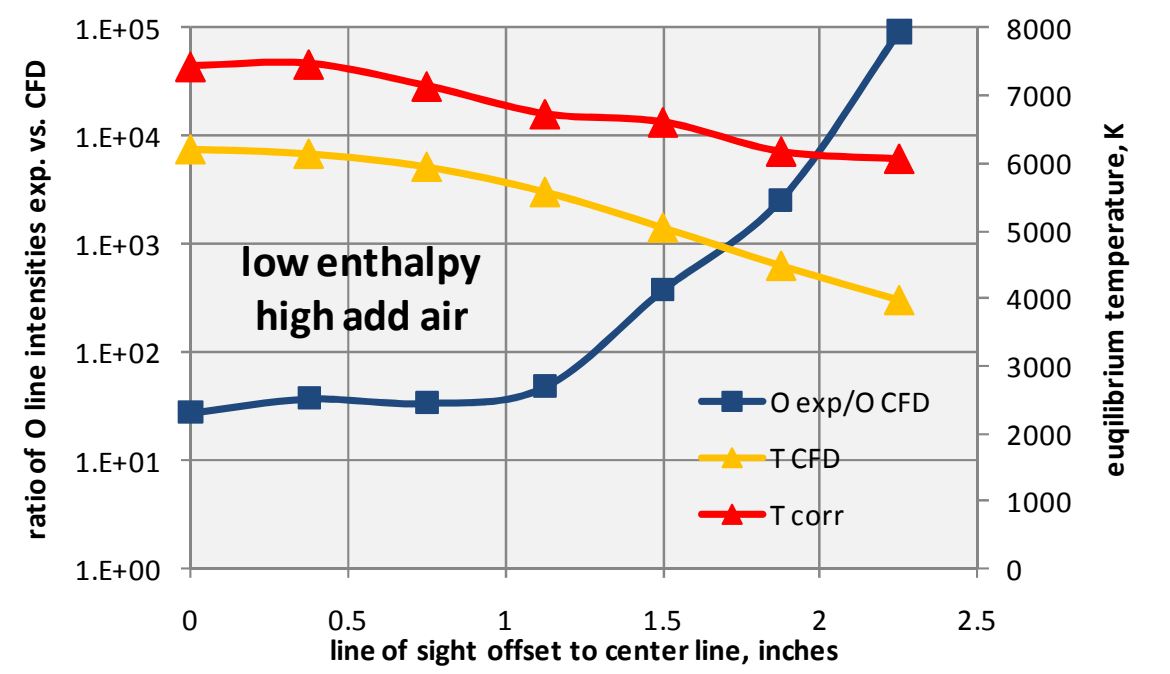

Figure 13. Oxygen line intensity ratio between CFD and experiment, CFD temperature, and corrected temperature vs. line of sight offset from center line.

\section{Conclusion}

An existing concept for optical measurements in the plenum region of the Ames arc-jet plasma heaters was modified to derive a design applicable to the IHF geometry. Optical fibers had to be used to access the inner region of the plasma heater. The final set-up involved a lab spectrometer and a set of optical fibers which are re-oriented to a linear array to enable a simultaneous measurement of all seven radial positions inside the plenum with the same spectrometer/CCD combination. The set-up was successfully tested at two different heater conditions at high and low enthalpy and with minimum and high flow rates of added air, respectively. In the core flow, the measurements at high enthalpy and minimum add air were in reasonable agreement with CFD based simulated spectra. At higher off set values, the radial profiles of the oxygen and nitrogen line intensities showed larger values for the predicted spectra, suggesting an over-prediction of plenum temperature by $400-800 \mathrm{~K}$ close to the wall.

At low enthalpy and high flow rate of added air, the measured spectra clearly indicate that the plasma temperature is under-estimated by the current staring condition for the CFD computation. The ratio between measured and simulated oxygen emission was used to estimate a necessary temperature correction for this starting condition to match the measured profiles yielding a temperature increase of the order of $1200 \mathrm{~K}$ to $2000 \mathrm{~K}$ depending on the radial position. This indicates that the mixing process is not adequately addressed in CFD.

These preliminary results will be checked by a systematic variation of enthalpy profile and, if necessary, also bulk enthalpy, to match the measured spectra. This task will be repeated for as many heater conditions as possible to create a data base for the operational envelope of the IHF facility for further investigation. One possible way to account for the differences between simulation and experiment seen in this work would be to establish emission spectroscopy measurements in the plenum as a standard survey of the plenum region. The results could be used as an input to the flow solver. Finally, the proposed processes will have to be correlated with free stream optical diagnostics and calorimetric measurements. In addition, optical measurements as a part of each arcjet run would allow for a verification of the conditions after any modification of the hardware (e.g. plenum package changes) as part of the standard facility calibration/operation procedure. 


\section{Acknowledgments}

The present work was supported by NASA Contract NAS2-03/44 to UARC, UC Santa Cruz, by NASA Contract NNA04BC25C to ELORET Corporation,and by the NASA-SCAP (Strategic Capabilities Assets Program) which provides critical financial support of the arc jet operational capability at Ames. The authors would like to thank Dr. George Raiche (Chief, Thermophysics Facilities Branch, NASA ARC) for generously providing test time in the IHF facility for the experiment, and Dr. Aga Goodsell (Chief, Reacting Flow Environments Branch, NASA ARC) for support of modeling and simulation aspects of the present work. Furthermore, the authors wish to acknowledge the support of Jay Grinstead, Vince Meglio, Enrique Carballo, Corinna Thompson and thank the arc jet crew for their professional and tireless effort to maintain and operate the arc jets under demanding schedule pressures.

\section{References}

${ }^{1}$ Prabhu, D.K., Saunders, D.S., Oishi, T., Skokova, K., Santos, J., Fu, J., Terrazas-Salinas, I., Carballo, E., and Driver, D.M., "CFD Analysis Framework for Arc-Heated Flowfields, I: Stagnation Testing in Arc-jets at NASA ARC," AIAA Paper AIAA2009-4080, $41^{\text {st }}$ Thermophysics Conference, June 22-25, 2009, San Antonio, TX.

${ }^{2}$ Taunk, J.S., Shepard, C.E., and Carrasco, A. "Design and Verification of a Copper Disk for Radiation Measurement in the Constrictor Region of an Arcjet”, ISA Paper No. 94-3025, Baltimore, MD, (1994).

${ }^{3}$ Donohue, J.M., Fletcher, D.G., Park, C.S., "Emission Spectral Measurements in the Plenum of an Arc-Jet Wind Tunnel," AIAA Paper No. 98-2946, 7th AIAA/ASME Joint Thermophysics and Heat Transfer Conference, June, (1998).

4 Space Technology Division, NASA Ames Research Center, Moffett Field, CA 94035. http://thermophysics.arc.nasa.gov/fact sheets/ArcJet\%20Fact\%20Sheet.pdf

${ }^{5}$ Wright, M.W., White, T., and Mangini, N., "Data Parallel Line Relaxation (DPLR) Code User Manual Acadia - Version 4.01.1," NASA/TM-2009-215388, October 2009..

${ }^{6}$ Whiting, E.E., Park, C., Liu, Y., Arnold, J.O., and Paterson, J.A., "NEQAIR96, Nonequilibrium and Equilibrium Radiative Transport and Spectra Program: User's Manual," NASA RP-1389, NASA, December 1996. 